\title{
EXPLORANDO O PROGRAMA DE VEÍCULOS AÉREOS NÃO-TRIPULADOS DA CHINA
}

\author{
EXPLORING THE AIR VEHICLES PROGRAM UNMANNED OF CHINA
}

Fernando Marcelino Pereira ${ }^{1}$

\begin{abstract}
RESUMO
0 presente artigo analisa o programa de desenvolvimento de drones da China. Debatemos o impacto estratégico dos drones no sistema internacional pós-guerra fria e os esforços que a China vem fazendo para desenvolver essa tecnologia. Concluímos que o programa chinês está se desenvolvendo rapidamente porque conta com a sinergia do setor militar-industrial, centros de pesquisa, capacitação técnica, financiamento e infra-estrutura.
\end{abstract}

Palavras-chave: China; segurança; drones.

\begin{abstract}
This article analyzes the drone development program of China. We discussed the strategic impact of drones in the post-Cold War international system and the efforts that China has been doing to develop this technology. We conclude that the Chinese program is developing rapidly that it relies on the synergy of the military-industrial sector, research centers, technical training, finance and infrastructure.
\end{abstract}

Key-words: China; security; drones.

\section{INTRODUÇÃO}

Os Veículos Aéreos Não Tripulados (VANTS), ou simplesmente drones, têm sido objeto de diversas discussões sobre o futuro dos conflitos internacionais. Esses equipamentos estão alterando a forma como as guerras são combatidas e passam a ser alvo de uma intensa corrida tecnológica. Apesar da hegemonia dos Estados Unidos na área, que vem usando drones de forma sistemática e contínua em campo de batalha

${ }^{1}$ Graduado em Relações Internacionais pela UNICURITIBA, Mestre em Ciência Política e Doutorando em Ciências Sociais na Universidade Federal do Paraná - UFPR. Consultor especializado em China.

Conjuntura Global, vol. 5 n. 1, jan./abr., 2016, p. 51-64. 
desde 2002, outros países também estão desenvolvendo tecnologias na área. Neste artigo vamos analisar como a China tem delineando políticas e desenvolvido um dos maiores e mais complexos programas de drones no mundo. Este trabalho se baseia em leituras de trabalhos e pesquisas, notícias da mídia chinesa e internacional, contato com fabricantes estatais e privados de drones chineses, participação em debates na Universidade de Pequim e coleta de informações em 2015 na China Aviation Expo em Pequim e a International UAV Technology \& Equipment Exhibition em Shangai, as duas maiores feiras especializadas em drones da China de 2015.

\section{DRONES NA SEGURANÇA INTERNACIONAL PÓS-GUERRA FRIA}

As questões relativas à "segurança" tomam uma dimensão cada vez mais central na resolução dos conflitos nacionais e internacionais na atualidade. Desde o início do contexto pós-guerra fria no final dos anos 1980, a noção de "segurança" tomou uma proporção cada vez maior para organismos internacionais e para os Estados nacionais ao englobar uma enorme diversidade de processos além da clássica preocupação com a soberania das nações: segurança energética, segurança migratória, segurança urbana, segurança alimentar, biossegurança, segurança humanitária, cibersegurança, segurança ecológica, segurança das propriedades intelectuais e genéticas, segurança privada, etc. É possível afirmar que uma das principais marcas desse período é uma generalizada securitização desigual do sistema mundial da dimensão mais micro (biogenética e cibernética) à macro (órgãos de defesa e segurança internacional, atividades extramilitares em diversos países, satélites de segurança). A agenda de segurança ampliou-se, agregando outras questões além da abordagem essencialmente militarizada. O inimigo, claramente definido no período de mais de quatro décadas da Guerra Fria, já não é mais tão facilmente identificável, e essa dificuldade impõe uma mudança na capacidade analítica e reativa dos Estados em se adequarem às novas ameaças contemporâneas ${ }^{2}$.

\footnotetext{
${ }^{2}$ Sobre os novos marcos da segurança internacional pós-guerra fria ver: Marcelino, Fernando. Desafios na formulação de uma estratégia comum de defesa regional na América do Sul: Enfoque na questão das empresas militares privadas e na Geopolítica Colombiana. Revista Relações Internacionais no Mundo Atual, Curitiba, v. 2, n. 14, 2011.
}

Conjuntura Global, vol. 5 n. 1, jan./abr., 2016, p. 51-64. 
Com o aprimoramento das tecnologias da informação e da comunicação, tornouse possível o desenvolvimento de sistemas de armas que permitem a manutenção da vigilância do território inimigo de forma precisa. Esse é o caso dos Veículos Aéreos Não Tripulados, ou drones, sistemas de vigilância com plataforma de ataque acoplada e que vêm sendo continuadamente empregados nas operações militares dos Estados Unidos contra movimentos insurgentes na fronteira entre Afeganistão e Paquistão especialmente em Waziristão Norte e Sul - desde meados de 2004, durante a administração de George W. Bush. Desde então, mais de 370 ataques foram realizados provocando mais de 3000 vítimas, dentre elas mais de $22 \%$ seriam civis e crianças e quase $80 \%$ são supostamente militantes, em que não é clara a definição se são militantes ou civis. Desse total, pouco mais de 50 pessoas seriam líderes militantes identificados pela CIA e pelo governo americano enquanto perfis perigosos e passíveis de eliminação. Apesar da dificuldade em obter esses dados e distinguir qual é a real amplitude de civis e militantes mortos, todas as organizações comprometidas com esses estudos são unânimes em afirmar que esses ataques atingiram o seu ápice durante a administração Obama, em 2009. Nesse período, mais de 330 ataques foram realizados, alcançando o pico de 122 no ano de $2010^{3}$.

O investimento estadunidense em drones tem crescido substancialmente ao longo dos últimos anos. 0 montante investido em 2001 foi de 667 milhões de dólares e o inventário de drones era de 167. Já em 2012 o montante requerido pelo Departamento de Defesa para compra e investimento foi de 3,9 bilhões de dólares, ao passo que o patrimônio subiu para aproximadamente 7.500 aeronaves, aproximando-se do montante de aeronaves pilotadas manualmente, 10.767. Em 2015, os EUA chegaram a 7.494 aviões não-tripulados ${ }^{4}$.

Os dois mais importantes drones estadunidenses contam com capacidades de ataque e de vigilância. Com asas em "V" invertido, o MQ-1 Predator pode operar em torno de 10.000 a 15.000 pés de altitude para adquirir as melhores imagens de seu sistema de câmeras, conta com o alcance de voo de 500 milhas náuticas de sua base e com a autonomia de 24 horas de voo. 0 MQ-9 Reaper pode alcançar até 50.000 pés de

\footnotetext{
${ }^{3}$ Serle, Jack. Drone Warfare: More than 2,400 dead as Obama's drone campaign marks five years. 2014. Disponível em: http://www. thebureauinvestigates.com/2014/01/23/more-than-2400-dead-as-obamasdrone-campaign-marks-fve-years/

${ }^{4}$ Chow, Denise. Drone Wars: Pilots Reveal Debilitating Stress Beyond Virtual Battlefeld. 2013. Disponível em: http://www.livescience.com/40959- military-drone-war-psychology.html

Conjuntura Global, vol. 5 n. 1, jan./abr., 2016, p. 51-64. 
altitude, um alcance de 2000 milhas náuticas, e 32 horas de autonomia de voo. Enquanto o primeiro é capaz de carregar dois mísseis "Hellfire", o segundo é capaz de carregar a mesma quantidade desses mísseis que um helicóptero Apache, ou seja, 16 unidades. Ambos são equipados com duas câmeras eletro óticas e uma com infra-vermelho, além de um sistema de targeting multi-espectral a laser capaz de detectar objetos em movimento. Esses drones são parte de um conjunto de sistemas que envolvem, ainda, uma estação de controle em terra e um link de satélite, uma vez que há radares incorporados nos sistemas desses drones $^{5}$.

Apesar do domínio principal dos EUA sobre os drones, existe um aumento relativo do resto do mundo neste mercado, uma vez que mais de 50 países estão atualmente a desenvolver drones, em comparação com mais de 70 países que já adquiriram vários tipos de drones. Na verdade, um dos fatos mais alarmantes para os Estados Unidos e a supremacia de sua política de drones é que a China, Rússia e outras potências vêm desenvolvendo rapidamente a tecnologia de drones e outros veículos nãotripulados. As vantagens dos Estados Unidos vêm sendo progressivamente negadas com o desenvolvimento de uma nova geração de drones. Porém, enquanto o programa de drones dos Estados Unidos recebe muita atenção e estudo, o programa chinês continua relativamente desconhecido, especialmente no Brasil.

\section{O PROGRAMA DE DRONES CHINESES}

A indústria chinesa de drones se origina nos anos 1950 a partir de veículos estrangeiros:

- A União Soviética proveu drones La-17 para localização de alvos. Este drone serviu para que a Força Aérea da China viesse a produzir o drone Chang Kong-1 que fez seu primeiro vôo no dia 6 de dezembro de 1966.

- 0 Exército da China capturou um drone AQM-34 Firebee dos Estados Unidos no Vietnam do Norte dos anos 1960. Com engenharia reversa os chineses construíram o drone de baixa-altitude Wu Zhen-5 (WZ-5).

\footnotetext{
${ }^{5}$ Gertler, Jeremiah. U.S. Unmanned Aerial Systems. Washington DC, Congressional Research Service, 2012.
} 
A partir dos anos 1980, a China começou a investir em tecnologias próprias, a exceção do drone Harpy anti-radiação que foi comprado de Israel. Desde 1990 a China está engajada numa ampla modernização militar com objetivo de impedir forças externas em conflitos regionais, dar suporte a diplomacia chinesa em territórios marítimos em disputa e conduzir outros tipos de operação militar que projete a China em seus diversificados e crescentes interesses globais ${ }^{6}$.

Desde então, as Forças Armadas da China estão rapidamente se tornando uma organização militar de alta tecnologia muito sofisticada, que está focada no desenvolvimento de capacidades de guerra assimétrica que lhe permitam derrotar os Estados Unidos ou outras forças armadas avançadas em um conflito futuro ${ }^{7}$. Os novos recursos incluem armas anti-satélites, incluindo um míssil que orbita a terra com capacidade de atingir satélites estratégicos tão alto quanto 22.000 milhas no espaço, supercomputadores de guerra cibernética, sistemas de mísseis ofensivos, incluindo veículos hipersônicos e pela emergência de uma variedade de novas plataformas, dentre elas os veículos aéreos não-tripulados, veículos marítimos não-tripulados e navios de superfície não-tripulados ${ }^{8}$.

Diferentes métodos para captar novas tecnologias têm sido utilizados, como engenharia reversa (que tem sido dominante), visitas a experts em drones nos Estados Unidos, espionagem pela unidade 61389 do Exército $^{9}$ e contratos internacionais para obtenção de tecnologia.

Na parada militar de 2009, o Exército mostrou o BZK-006, drone tático de reconhecimento. Este drone peso-leve de médio alcance transporta uma pequena torre debaixo do nariz abrigando as câmeras FLIR/CCD para missões de dia/noite. Ele também pode levar um pequeno radar de vigilância terrestre com uma antena que fornece o datalink em tempo real entre o drone e a estação de comando em terra. Cada estação pode controlar dois drones ao mesmo tempo. BZK-006 é alimentado por um

\footnotetext{
${ }^{6}$ Easton, Ian M., and L. C. Russell Hsiao, “The Chinese People's Liberation Army's Unmanned Aerial Vehicle Project: Organizational Capacities and Operational Capabilities," Project 2049 Institute, March 2013. As of December 29, 2014: http://project2049.net/documents/uav_easton_hsiao.pdf

${ }^{7}$ Liu Yuan-chung, "Applications, Development of PLA UAV," Chueh-chi Tung-ya, September 1, 2009.

${ }^{8}$ Hsu, Kimberly, "China's Military Unmanned Aerial Vehicle Industry," U.S.-China Economic and Security Review Commission, June 2013.

9 Esta unidade também é conhecida como Instituto de Pesquisa GSD 55 ou Instituto de Informação Tecnológica de Beijing [北京信息技术研究所].

Conjuntura Global, vol. 5 n. 1, jan./abr., 2016, p. 51-64. 
motor a pistão de 4 cilindros, tem $4.3 \mathrm{~m}$ de comprimento, 1,5 m de altura, resistência $12 \mathrm{hr}$. Já na parada militar de 2015, foi apresentado o BZK-008, com uma torre retrátil com câmera CCD para missões dia e noite. Devido ao seu curto alcance, a informação recolhida é transmitida diretamente de volta para a estação terrestre. É menor e com maior capacidade que seu antecessor. Tem 3,5 metros de comprimento, altura 1,4 milhões, asa extensão $4.4 \mathrm{~m}$, peso de $110 \mathrm{~kg}, 20 \mathrm{~kg}$ de carga útil, resistência 5HR, 4,480m teto e velocidade máxima $180 \mathrm{~km} / \mathrm{h}^{10}$.

Em 2013 foi realizado o vôo inaugural do "Sharp Sword", drone desenvolvido conjuntamente pela Aviation Industry Corporation of China, Shenyang Aerospace University e Hongdu Aviation Industry Group. 0 drone é alimentado por um motor RD93 turbofan russo tem uma envergadura de 14 metros, muito parecido com o drone estadunidense X-47B desenvolvido pela Northrop Grumman. Feita a partir de tungstênio e outros materiais compósitos, a missão principal é reconhecimento de longo alcance, mas também pode ser implantado para o combate, salvamento e missões anti-pirataria e anti-terrorismo.

Outros importantes drones chineses são o Xianglong de reconhecimento em alta altitude e alta velocidade, semelhante ao do estadunidense Global Hawk, o drone Anjian, que é muito semelhante a um bombardeiro Stealth e o Pterodactyl (Yilong), que se assemelha ao drone Predator dos EUA ${ }^{11}$.

No final de 2014 a China começou a produção em série do drone armado Caihong4 cujas características operacionais o permitem lançar armas as altitudes de 16400 pés. Em geral, os drones equivalentes só podem lançar armas a um teto máximo de 10.000 pés. O Caihong-4 possui aerodinâmica e propulsores que lhe dá grande importância tática em termos de segurança e ataque instantâneo após a detecção de um alvo, algo que os drones ocidentais armados com o mísseis Hellfire não conseguem fazer.

Outro drone lançado em 2014 é o WJ-600 de alta altitude e velocidade. É um grande drone como um míssil de cruzeiro, com vários equipamentos de reconhecimento

10 Fisher, Richard. "China Seeks UAV Capability." Aviation Week. 1 Jul 2011. http://aviationweek.com/awin/china-seeks-uav-capability.

${ }^{11}$ Sobre os novos modelos de drones chineses ver "Remote control war Unmanned combat air vehicles in China, India, Iran, Israel, Russia and Turkey" organizada por Rob O'Gorman e Chris Abbott. Open Briefing. 2013.

Conjuntura Global, vol. 5 n. 1, jan./abr., 2016, p. 51-64. 
eletrônico avançado e um radar de abertura sintética. Ele também pode transportar dois mísseis KD-2.

Em 2015 os chineses apresentaram o drone $\mathrm{CH}-4$, com tecnologia via satélite, um drone de seis rotores que foi comparado ao Predator dos militares dos EUA. Além de poder atacar alvos localizados 5 mil metros abaixo, ele também pode realizar atividades de reconhecimento e fazer pesquisa mineral e geológica. Suas habilidades são equivalentes ao mais recente drone do exército dos EUA. O CH-4 pode levar 4-6 mísseis, seu corpo, rotores e trem de pouso são construídos de um composto de fibra de carbono, tem alcance operacional de $3.500 \mathrm{~km}$ e capacidade de vôo de 40 horas. Ele também é capaz de decolar verticalmente.

Em 2015 os chineses também lançaram sua própria versão do MQ-9 Reaper. The Wing Loong II foi apresentada na China Aviation Expo 2015 e tem atraído a atenção devido a sua semelhança com o Reaper. Ambos são movidos por um único motor turboélice empurrador, com sistema de comunicação por satélite e sistema óptico primário no nariz. No entanto, o teto operacional máximo do Wing Loong II e a velocidade é significativamente menor do que o Reaper: enquanto o MQ-9 tem uma velocidade máxima de $444 \mathrm{kmh}$ e pode atingir uma altitude máxima de 50.000 pés, o Wing Loong II tem uma velocidade máxima de $370 \mathrm{~km} / \mathrm{h}$ e pode atingir uma altitude de 30.000 pés $(9.000 \mathrm{~m})$. Além disso, o Reaper pode transportar até 3.750 libras (1.701 quilos) de portaria, enquanto o Wing Loong II só pode ser equipado com até $£ 1.050$ (480 quilogramas). Porém, o Wing Loong II é significativamente mais avançando que seu predecessor, inclusive com maior poder de fogo ${ }^{12}$.

Esta múltipla capacidade de operação dos drones chineses demonstra que a indústria de drones vem se desenvolvendo rapidamente. Muitos analistas presumem uma estrutura centralmente regulada, mas o programa de drones chinês oferece um mix de suporte financeiro que permite significativa dispersão e variação na produção de drones. A descentralização do programa chinês opera com abundância de atores que permite que diferentes níveis de tecnologia sejam desenvolvidos simultaneamente. A indústria de drones chinesa é composta por centenas de firmas e grupos de pesquisa,

12 Ramsley, Adam. Meet China's Killer Drones. Fereign Policy. Janeiro, 2016. Web. http://www.foreignpolicy.com/2016/01/14/meet-chinas-killer-drones. 
empresas ligadas ao Exército, indústrias estatais, universidades e setor privado ${ }^{13}$. Essa combinação de entidades representa os esforços de integração civil-militar com centros universitários de pesquisa, principalmente a Beijing University of Aeronautics and Astronautics, Nanjing University for Aeronautics and Astronautics e a Northwest Polytechnical University's Xi'an ASN Technology Group. Também existem numerosos centros de pesquisa do Exército associados à indústria de defesa, como a Chengdu Aircraft Industry Group/Chengdu Aircraft Design Institute, Guizhou Aircraft Industry Corporation, Shenyang Aircraft Company/Shenyang Aircraft Design Institute, China Aerospace Science and Technology Corporation e a Nanjing Research Institute on Simulation Technique. Para completar, praticamente todas as maiores indústrias de armas da China tem centros de pesquisas sobre drones, o que demonstra um complexo industrial diversificado que estimula a competição e o rápido desenvolvimento tecnológico, o que deve aumentar significativamente o número e a qualidade dos drones chineses ${ }^{14}$.

\section{MOTIVAÇÕES DO PROGRAMA CHINÊS}

O programa de drones chinês tem motivações próprias. Em primeiro lugar, possibilitar que as Forças Armadas alcancem missões de ataques precisas com raio de $3.000 \mathrm{~km}$ da costa da China. A China tem utilizado aviões não tripulados para vigilância nos territórios em disputa no Mar do Sul da China e inclusive já admitiu voar um drone BZK-005 perto das ilhas Senkaku disputadas com o Japão. 0 complexo militar-industrial da China criou na última década uma ampla gama de aviões não-tripulados para lograr esse objetivo. Em segundo lugar, os chineses usam drones para vigilância de movimentos

\footnotetext{
${ }^{13}$ Um exemplo de drone desenvolvido no setor privado é o EHang, um drone capaz de transportar um passageiro, ou carga de até $100 \mathrm{~kg}$. 0 veículo, chamado EHang 184 , mede cerca de $1,5 \mathrm{~m}$, pesa $200 \mathrm{~kg}$ e consegue atingir velocidade de até $100 \mathrm{~km} / \mathrm{h}$. Para se deslocar, basta abrir o aplicativo para smartphone e indicar seu local de destino. 0 drone pode voar a uma altura de 11 mil pés (cerca de $3,3 \mathrm{~km}$ ) e, de acordo, com a companhia, funciona por até 23 minutos. Depois de zerada, a bateria pode ser recarregada completamente em 4 horas. Foi apresentado no início de 2016. É possível que drones como este sejam desenvolvidos com objetivos militares no futuro próximo.
}

${ }^{14}$ Erickson, Andrew \& Austin Strange. "China Has Drones. Now What?" Foreign Affairs. Council on Foreign Relations, 2013. Web. http://www.foreignaffairs.com/articles/139405/andrew-erickson-and-austinstrange/china-has-drones-now-what.

Conjuntura Global, vol. 5 n. 1, jan./abr., 2016, p. 51-64. 
terroristas dentro do país, especialmente na província de Xinjiang. Em terceiro lugar, os chineses também começaram a usar drones para reconhecimento e para guiar seus mísseis balísticos anti-navais DF-21D, o que irá garantir grande parte do controle sobre as águas do Mar da China Meridional, o restante sendo por controlado por porta-aviões chineses. Dessa forma, a China está construindo uma das maiores frotas de drones do mundo para ampliar o seu alcance militar no Pacífico, conter a crescente presença dos Estados Unidos no Pacífico e desafiar seus porta-aviões em caso de guerra15.

Atualmente as Forças Armadas da China empregam drones numa série de missões, integrando exercícios de treinamento, vigilância, unidades de controle e centro de comunicação. Um levantamento preliminar de unidades prováveis indica que os drones estão espalhados por todos os ramos do serviço Exército. Quanto à plataforma utilizada pelo Exército, existem os seguintes tipos de missões principais: inteligência, vigilância e reconhecimento; missões com drones equipados com material eletro-óptico; radar, sensores eletrônicos e identificação de sinais; localização, rastreamento e segmentação porta-aviões norte-americanos avaliação de danos de batalha; missões de ataque de precisão; análise de radiação; missões de guerra eletrônica e missões de retransmissão de dados $^{16}$. Também existem missões para objetivos não-militares, incluindo vigilância marítima, mapeamento agrícola, assistência humanitária e desastres naturais como terremotos. Nas missões de longo alcance, os drones podem estender o horizonte de alvos como mísseis balísticos, artilharia DF-21D e foguetes anti-submarino. Em particular, os drones podem são importantes em detectar, localizar e apontar alvos fixos e em movimento - como navios e submarinos dos Estados Unidos. Nas missões de médio alcance, os drones podem fixar alvos em Taiwan e navios em sua costa17.

\footnotetext{
15 Rasmussen, Ivan Willis. Everyone Loves Drones, Especially China Exploring the motivations of the Chinese UAV program. Conflict, Security, and Public Policy Working Group Belfer Center for Science and International Affairs, Harvard Kennedy School, 2014.
}

\footnotetext{
${ }^{16}$ A indústria de drones chinesa vem desenvolvendo drones especiais para guerra eletrônica. Eles são de alta altitude e podem dissuadir satélites de comunicação e global positioning system (GPS), inclusive com a geração de alvos falsos de mísseis inimigos.

${ }^{17}$ Xiong, Feng, et al., "Research on Using UAV Data Link Technology to Elevate Anti-Ship Missile Attack Capability” [“利用无人机中 继指导技术提高反舰导弹攻击能力研究”], Winged Missile Journal (Feihang Daodan) [飞航导弹], No. 8, 2004, pp. 35-37.

Conjuntura Global, vol. 5 n. 1, jan./abr., 2016, p. 51-64. 
Os chineses têm planos de produzir mais de 41.800 sistemas não-tripulados terrestres e marítimos, no valor de cerca de US\$10,5 bilhões entre 2014 e $2023^{18}$.

Uma rede robusta de drones, combinado com satélites, navios e jatos de ponta irá tornar o Exército da China cada vez mais capaz de localizar frotas adversárias em distâncias maiores, e, uma vez descoberto, monitorá-los continuamente. 0 Exército já vem desenvolvendo, inclusive, drones que podem voar em formação, participar de reabastecimento aéreo e decolagem e de terras de forma autônoma. Engenheiros do Exército também começaram a explorar a utilização de aeronaves tripuladas para controlar drones, o que deve estar ligado a uma ambição de desenvolver drones de longo alcance para bombardeio. Segundo a Academia de Engenharia Física da China, engenheiros chineses desenvolveram com sucesso uma arma a laser que consegue destruir drones que circulem abaixo dos 500 metros de altitude e numa velocidade não superior a 180 quilômetros por hora. O sistema de baixa altitude Sentinel consegue detectar pequenos objetos voadores num raio de dois quilômetros e disparar contra deles em cinco segundos. Pode ser instalado em carros e permite às forças segurança a proteção contra este tipo de objetos voadores em grandes eventos nas áreas urbanas. Em 2012 foi anunciada a criação de duas primeiras bases de drones na província de Liaoning para monitorar a situação em águas costeiras. No final de 2015 já eram 11 bases de monitoramento de drones ${ }^{19}$.

\section{O PAPEL DOS DRONES NO PLANO MADE IN CHINA 2025}

Em 19 de maio de 2015, o Conselho de Estado da China lançou o Plano "Made In China 2025", visando ser a primeira fase da estratégia de 30 anos para transformar seu setor manufatureiro até 2049, ano que marca o 100 aniversário da fundação da República Popular da China.

18 "China planning to build 42,000 drones, report says". Por William Lowther. Taipei Times. 10 de maio de 2015.

19 Keck, Zachary. "China to Lead World in Drone Production." The Diplomat. 2 May 2014. Web. http://thediplomat.com/2014/05/china-to-lead-world-in-drone-production/.

Conjuntura Global, vol. 5 n. 1, jan./abr., 2016, p. 51-64. 
O objetivo é atualizar de forma abrangente a indústria chinesa, tornando-a mais eficiente e integrada para que possa ocupar a maior parte das cadeias de produção globais. 0 plano identifica o objetivo de elevar o conteúdo nacional de componentes e materiais a 40\% em 2020 e 70\% até 2025. Embora haja um papel significativo para o Estado em proporcionar um quadro global, utilizando instrumentos financeiros e fiscais, e apoiar a criação de fabricação de centros de inovação (15 em 2020 e 40 em 2025), o plano também conta com instituições de mercado, reforço da proteção direitos de propriedade intelectual para as pequenas e médias empresas (PME) e de uma utilização mais eficaz da propriedade intelectual (IP). O Plano foi elaborado "em linha com os rápidos avanços na ciência e na tecnologia" para promover "avanços em dez setoreschave": 1) Nova tecnologia de informação avançada; 2) automatizado máquinasferramentas e robótica; 3) Drones e equipamentos aeroespaciais; 4) Equipamento de transporte marítimo e de alta tecnologia; 5) equipamento de transporte ferroviário moderno; 6) Os veículos da nova energia e equipamentos; 7) Equipamento de alimentação; 8) Equipamento Agrícola; 9) Os novos materiais; e 10) Biofarma e produtos médicos avançados ${ }^{20}$.

A inauguração do "Made in China 2025" sob o governo Xi Jinping sugere uma ruptura importante com a abordagem do governo anterior Hu-Wen à inovação e modernização tecnológica. 0 coração de sua abordagem foi o Plano de Médio e Longo Prazo para o Desenvolvimento da Ciência e Tecnologia. Um plano de 15 anos emitido em 2006, conceito-chave do plano era "inovação nativa" (自主 创新) e focado inteiramente em tecnologias avançadas. 0 ponto culminante do plano foi a identificação em Outubro de 2010 de sete "indústrias estratégicas emergentes" (战略性 新兴产业) que eram vistos como vitais para a China para conseguir o domínio em se era para se tornar uma economia avançada. 0 núcleo do plano focado em tecnologias de ponta avançadas desenvolvidas. O "Made in China 2025" é diferente em pelo menos três aspectos: 1) Incide sobre todo o processo de fabricação e não apenas a inovação; 2) Promove o desenvolvimento não apenas de indústrias avançadas, mas as indústrias tradicionais e

\footnotetext{
20 'Made in China 2025' plan unveiled, Xinhua, 19/05/2015. Disponível em: http://www.chinadaily.com.cn/bizchina/2015-05/19/content_20760528.htm 
serviços modernos; 3) Ainda há um foco no envolvimento do Estado, mas os mecanismos de mercado são mais proeminentes ${ }^{21}$.

Neste Plano, o desenvolvimento dos drones passa a ser um "setor-chave" para se beneficiar de um financiamento significativo e apoio específico por parte do governo da China para as próximas décadas. 0 plano Made In China 2025 prevê um aumento dos empréstimos por bancos públicos e bancos comerciais para drones e outros setoreschave, o aumento do financiamento de pesquisas e a adoção de políticas fiscais preferenciais para estimular ainda mais o crescimento do setor. Empresas estatais de defesa já começaram a alocar recursos substanciais e conhecimentos de engenharia para o desenvolvimento de drones comerciais em segmentos chave do mercado, incluindo mapeamento aéreo, levantamento de recursos e de gasodutos, monitorização da rede, agricultura, mídia, seguros, imobiliário, serviços públicos, entre muitos outros. 0 Made In China 2025 estabelece o desenvolvimento de drones como um motor essencial da economia, com metas claras e múltiplas ferramentas de suporte financeiro para efetuar essas metas, incluindo empréstimos do governo e de políticas fiscais preferenciais. Este programa para financiar de forma agressiva e crescente da indústria drones chinês deve ter conseqüências profundas para os fabricantes drones e indústrias associadas em todo o mundo.

\section{Considerações finais}

A China tem desenvolvido um dos maiores e mais complexos programas de drones no mundo e está se aproximando rapidamente dos Estados Unidos na corrida pelo desenvolvimento da tecnologia, redefinindo as condições da concorrência global na área. Este programa inclui um enorme projeto militar-industrial, centros de pesquisa universitários e militar, financiamento, infra-estrutura e um número crescente de drones operacionais espalhados por todos os ramos das forças armadas chinesas. Isso mostra que os drones são cada vez mais estratégicos na projeção militar da China, o que seguramente tem impactos significativos na segurança regional asiática nos próximos

\footnotetext{
${ }^{21}$ Stewart, Phil. "Chinese military spending exceeds $\$ 145$ billion, drones advanced: U.S." Reuters. 6 Jun $2014 . \quad$ Web. http://www.reuters.com/article/2014/06/06/us-usa-china-militaryidUSKBNOEG2XK20140606.
}

Conjuntura Global, vol. 5 n. 1, jan./abr., 2016, p. 51-64. 
anos e pode ser um importante elemento na construção na nova balança de poder geopolítico global.

O governo chinês considera os drones uma tecnologia chave em seu desenvolvimento militar. Apesar da discrição, o Exército chinês identifica em seu programa de drones uma forma de reduzir ou eliminar vantagens tecnológicas dos Estados Unidos e considera-o um componente integral de guerra. Sua doutrina enfatiza o uso de armas do espectro eletromagnético para suprimir ou enganar o inimigo com equipamentos eletrônicos. A estratégia chinesa salienta que o uso de drones é uma dimensão para combate que pode ser vital para determinar o resultado de uma guerra, pois é uma força multiplicadora capaz de ser empregada em apoio a todas as armas e serviços de combate durante um conflito. Isso poderia colocar outros exércitos em clara desvantagem, especialmente se eles não têm as suas próprias capacidades de reconhecimento e vigilância de drones.

Dessa maneira, como visto, o desenvolvimento de drones pelos chineses representa profundas transformações na esfera de segurança, economia e política. Trata-se de avançar em novas tecnologias, novas capacidades militares, novas indústrias, cadeias produtivas e instrumentos assimétricos para defender sua soberania e suas posições políticas perante sua região e ao resto do mundo.

\section{REFERÊNCIAS}

Easton, Ian M. \& LC Russell Hsiao. "The Chinese People's Liberation Army's Unmanned Aerial Vehicle Project: Organizational Capacities and Operational Capabilities." Project 2049 Institute, 11 Mar 2013.

Erickson, Andrew, and Austin Strange. "China Has Drones. Now What?" Foreign Affairs. Council on Foreign Relations, 23 May 2013. Web. http://www.foreignaffairs.com/articles/139405/andrew-erickson-and-austin-strange/china-hasdrones-now-what.

Fisher, Richard. "China Seeks UAV Capability." Aviation Week. 1 Jul 2011. Web. http://aviationweek.com/awin/china-seeks-uav-capability. 
Gertler, Jeremiah. U.S. Unmanned Aerial Systems. Washington DC, Congressional Research Service, 2012.

Hannas, William C., James Mulvenon, and Anna B. Puglisi. Chinese Industrial Espionage: Technology Acquisition and Military Modernization. New York: Routledge, 2013.

Liu Yuan-chung, “Applications, Development of PLA UAV," Chueh-chi Tung-ya, September $1,2009$.

Keck, Zachary. "China to Lead World in Drone Production." The Diplomat. 2 May 2014. Web. http://thediplomat.com/2014/05/china-to-lead-world-in-drone-production/.

Marcelino, Fernando. Desafios na formulação de uma estratégia comum de defesa regional na América do Sul: Enfoque na questão das empresas militares privadas e na Geopolítica Colombiana. Revista Relações Internacionais no Mundo Atual, Curitiba, v. 2, n. 14, 2011.

Stewart, Phil. "Chinese military spending exceeds $\$ 145$ billion, drones advanced: U.S." Reuters. 6 Jun 2014. Web. http://www.reuters.com/article/2014/06/06/us-usa-chinamilitary-idUSKBNOEG2XK20140606.

Tai Ming Cheung. "Dragon on the Horizon: China's Defense Industrial Renaissance." Journal of Strategic Studies. Vol. 32.1, p. 29-66, 2009.

Ramsley, Adam. Meet China's Killer Drones. Fereign Policy. Janeiro, 2016. Web. http://www.foreignpolicy.com/2016/01/14/meet-chinas-killer-drones.

Wendell Minnick, "China developing armed/recon UAVs," Defense News, November 24, 2010. Web. http://www.defensenews.com/article/20101124/C4ISR01/11240301/Chinadeveloping-armed-recon-UAVs.

Whetham, David. "Killer Drones: the Moral Ups and Downs". In: The RUSI Journal, 158:03, pp. 22-32. 2013.

Wong, Edward. "Hacking U.S. Secrets, China Pushes for Drones." New York Times 20 Sep 2013. Web. http://www.nytimes.com/2013/09/21/world/asia/hacking-us-secrets-china-pushesfor-drones.html?_r=1\&.

Wong, Kelvin. "Armed Drones in Asia." S. Rajaratnam School of International Studies. 23 Aug 2010.

Xie Yibin; Chen Long, "Unmanned Jamming Vehicle Strikes," Renmin Qianxian (People's Front), May 10, 2012.

Conjuntura Global, vol. 5 n. 1, jan./abr., 2016, p. 51-64. 
Xu, Tianran. "Orders taken for Chinese drone." Global Times. 15 Nov 2012. Web. http://www.globaltimes.cn/content/744417.shtml. 